\title{
QUADRATIC FUNCTIONAL FOR SYSTEMS WITH DISTRIBUTED TIME DELAYS ${ }^{2}$
}

\author{
Omar Santos* V. L. Kharitonov* S. Mondié ${ }^{*, 1}$ \\ * Department of Automatic Control CINVESTAV-IPN \\ A.P. 14-740, Mexico, D.F. MEXICO \\ (osantos, Khar, smondie)@ctrl.cinvestav.mx
}

\begin{abstract}
Complete type Lyapunov Krasovskii functionals for systems with distributed time delays are presented. The derivative of these functionals depends on past states, and they admit a quadratic lower bound. These functionals are suitable for obtaining exponential estimates of the solution and for robustness analysis of uncertain systems Copyright (C)2005 IFAC.
\end{abstract}

Keywords: Lyapunov-Krasovskii functionals, time delay systems, exponential estimates, robust stability.

\section{INTRODUCTION}

Construction of Lyapunov-Krasovskii functionals with a given time derivative was initiated in (Repin, 1965). Since that several interesting results have been reported in (Datko, 1971), (Infante, 1978), (Huang, 1989). The main attention was paid to the case when the time derivative is a negative definite quadratic form of the present state of a system. As it has been demonstrated in (Huang, 1989) the corresponding functionals do not admit positive lower quadratic bounds, and therefore can not be applied, for example, for estimation of robustness of time delay systems. In a recent paper (Kharitonov, 2003), a modification of the functionals has been proposed that, for the case of exponentially stable systems, provides the modified functionals with positive quadratic lower bounds. The modified functionals were called as complete type ones. These complete type functionals, unlike the previous ones, have a time derivative that depends on the past states of the system. It has been demonstrated that the complete type functionals can be used for the de-

1 On leave at Heudiasyc, Compiègne, France

2 Partially supported by CONACyT, Proj. 41276-Y, Mex. termination of exponential estimates, and for the robust stability analysis of uncertain time delay systems.

In this contribution we extend the construction of complete type functionals, given in (Kharitonov, 2003), to the case of systems with distributed time delay. After introducing some preliminary results in Section 2, the construction of complete type functionals for systems with concentrated and distributed delays is addressed in Section 3. Then, in Section 4 and 5, it is shown how these functionals can be used to derive exponential estimates of the solutions of time delay systems, and to analyze the robust stability of uncertain systems. The paper ends with some concluding remarks.

\section{PRELIMINARIES}

Given a time delay system of the form

$$
\begin{aligned}
& \dot{x}(t)=\sum_{k=0}^{m} A_{k} x\left(t-h_{k}\right)+\int_{-\tau}^{0} Q(\theta) x(t+\theta) d \theta, \\
& \text { with } x(\zeta)=\varphi(\zeta), \text { for } \zeta \in[-H, 0],
\end{aligned}
$$


where $x(t) \in \mathbb{R}^{n}, A_{k} \in \mathbb{R}^{n \times n}, Q(\theta) \in \mathbb{R}^{n \times n}$ is defined for $\theta \in[-H, 0], 0<h_{1}<h_{2}<\ldots<h_{m}$, and $H=\max \left\{\tau, h_{m}\right\}$. Furthermore, each element of the matrix $Q(\theta)$ is continuous.

Definition 1. System (1) is said to be exponentially stable if there exist $\alpha>0$ and $\gamma \geq 1$ such that for every solution $x(t, \varphi)$ of system (1) with initial function $\varphi(\theta), \theta \in[-H, 0]$ the following condition holds

$$
\|x(t, \varphi)\| \leq \gamma\|\varphi\|_{H} e^{-\alpha t}, \quad \forall t \geq 0 .
$$

Here $\|\varphi\|_{H}$ denotes

$$
\|\varphi\|_{H}=\sup _{\zeta \in[-H, 0]}\|\varphi(\zeta)\|
$$

In this note, the term stable means exponentially stable. As it plays a central role in the construction of the complete type functional, the equivalent of the Cauchy formula for the solutions of system (1) is recalled.

Theorem 1. (Bellman and Cooke, 1963) Consider the $n \times n$ matrix function $K(t)$ such that the equation

$$
\begin{aligned}
\frac{d}{d t} K(t)= & A_{0} K(t)+\sum_{k=0}^{m} A_{k} K\left(t-h_{k}\right) \\
& +\int_{-\tau}^{0} Q(\theta) K(t+\theta) d \theta,
\end{aligned}
$$

with the initial condition $K(0)=I$, and $K(t)=0$ for $t<0$. Then for $t \geq 0$

$$
\begin{aligned}
x(t, \varphi)= & K(t) \varphi(0)+\sum_{k=0}^{m} \int_{-h_{k}}^{0} K\left(t-h_{k}-\zeta\right) A_{k} \varphi(\zeta) d \zeta \\
& +\int_{-\tau}^{0} \int_{\theta}^{0} K(t-\zeta+\theta) Q(\theta) \varphi(\zeta) d \zeta d \theta .
\end{aligned}
$$

The matrix $K(t)$ is know as the fundamental matrix of system (1) and each column of this matrix is a solution of the system. It also satisfies

$$
\begin{aligned}
\frac{d}{d t} K(t)= & K(t) A_{0}+\sum_{k=0}^{m} K\left(t-h_{k}\right) A_{k} \\
& +\int_{-\tau}^{0} K(t+\theta) Q(\theta) d \theta .
\end{aligned}
$$

If the system is stable then the matrix $K(t)$ also satisfies the inequality

$$
\|K(t)\| \leq \gamma e^{-\beta t}, \text { for all } t \geq 0 .
$$

Lemma 1. Let system (1) be stable, then for any $n \times n$ constant symmetric matrix $W$, the matrix

$$
U(\tau)=\int_{0}^{\infty} K^{T}(t) W K(t+\tau) d t
$$

is well defined for all $\tau \in \mathbb{R}$. It satisfies the

(i) symmetric property

$$
U(-\tau)=U^{T}(\tau), \text { for all } \tau \geq 0,
$$

(ii) dynamic property. For all $\tau \geq 0$,

$$
U^{\prime}(\tau)=\sum_{k=0}^{m} U\left(\tau-h_{k}\right) A_{k}+\int_{-\tau}^{0} U(\tau+\theta) Q(\theta) d \theta
$$

(iii) algebraic property

$$
-W=U^{\prime}(+0)-U^{\prime}(-0)
$$

\section{CONSTRUCTION OF COMPLETE TYPE FUNCTIONALS.}

We are looking for a Lyapunov-Krasovskii functional $v\left(x_{t}\right)$ such that

$$
\left.\frac{d v\left(x_{t}\right)}{d t}\right|_{(1)}=-w\left(x_{t}\right), \quad t \geq 0,
$$

where the functional $w\left(x_{t}\right)$ is of the form

$$
\begin{aligned}
w\left(x_{t}\right)= & x^{T}(t) W_{0} x(t)+\sum_{j=1}^{m} x^{T}\left(t-h_{j}\right) W_{j} x\left(t-h_{j}\right) \\
& +\sum_{k=1}^{m} \int_{-h_{k}}^{0} x^{T}(t+\theta) R_{k} x(t+\theta) d \theta \\
& +\int_{-\tau}^{0} x^{T}(t+\theta) M x(t+\theta) d \theta .
\end{aligned}
$$

Here $W_{k}, R_{k}, k=0, \ldots, m$, and $M$ are given positive definite matrices. Let $\varphi(\theta)$ be a continuous initial function on $[-H, 0]$. As the system is assumed to be stable, it follows that the functional $v\left(x_{t}\right)$ exists. Furthermore it can be written as

$$
v(\varphi)=\int_{0}^{\infty} w\left(x_{t}(\varphi)\right) d t
$$

This integral converges and is well defined. First, we build a functional $v_{0}\left(x_{t}\right)$ such that

$$
\frac{d}{d t} v_{0}\left(x_{t}\right)=-x^{T}(t) W x(t)
$$

Integrating this expression from 0 to $\infty$, and using the fact that the solution is stable yields

$$
v_{0}(\varphi)=\int_{0}^{\infty} x^{T}(t, \varphi) W x(t, \varphi) d t
$$

Substituting $x(t, \varphi)$ under the integral (11) by (3), and after some simple calculations we obtain 


$$
\begin{aligned}
& v_{0}\left(x_{t}\right)=x^{T}(t) U(0) x(t)+2 x^{T}(t) \times \\
& {\left[\sum_{j=1}^{m} \int_{-h_{j}}^{0} U\left(-h_{j}-\zeta\right) A_{j} x(t+\zeta) d \zeta\right.} \\
& \left.+\int_{-\tau}^{0} \int_{\theta}^{0} U(\theta-\zeta) Q(\theta) x(t+\zeta) d \zeta d \theta x(t)\right] \\
& +\sum_{j=1}^{m} \int_{-h_{j}}^{0}\left[\sum_{k=1}^{m} \int_{-h_{k}}^{0} x^{T}\left(t+\zeta_{1}\right) A_{k}^{T} \times\right. \\
& \left.U\left(h_{k}+\zeta_{1}-h_{j}-\zeta_{2}\right) A_{j} x\left(t+\zeta_{2}\right) d \zeta_{1}\right] d \zeta_{2} \\
& +2 \int_{-\tau}^{0} \int_{\theta}^{0} x^{T}\left(t+\zeta_{1}\right) Q^{T}(\theta) \times \\
& U\left(\zeta_{1}-\theta-h_{j}-\zeta_{2}\right) A_{j} x\left(t+\zeta_{2}\right) d \zeta_{1} d \theta \\
& +\int_{-\tau}^{0} \int_{\theta_{1}}^{0} \int_{-\tau}^{0} \int_{\theta_{2}}^{0} x^{T}\left(t+\zeta_{1}\right) Q^{T}\left(\theta_{1}\right) \times \\
& U\left(\zeta_{1}-\theta_{1}-\zeta_{2}+\theta_{2}\right) Q\left(\theta_{2}\right) x\left(t+\zeta_{2}\right) d \zeta_{2} d \theta_{2} d \zeta_{1} d \theta_{1},
\end{aligned}
$$

where the matrix $U(\tau)$ is defined by (6). Consider the next lemma.

Lemma 2. Given definite positive matrices $W_{j}$, $j=1,2, \ldots, 2 m$, and $M$. The time derivative of the functional

$$
\begin{aligned}
\tilde{v}\left(x_{t}\right)= & \sum_{j=1}^{m} \int_{-h_{j}}^{0} x^{T}(t+\theta)\left[W_{j}+\left(h_{j}+\theta\right) W_{m+j}\right] \times \\
& x(t+\theta) d \theta \\
& +\int_{-\tau}^{0}(\tau+\theta) x^{T}(t+\theta) M x(t+\theta) d \theta, \quad(13)
\end{aligned}
$$

is equal to

$$
\begin{aligned}
\frac{d \tilde{v}\left(x_{t}\right)}{d t}= & x^{T}(t)\left\{\sum_{j=1}^{m}\left(W_{j}+h_{j} W_{m+j}\right)+\tau M\right\} x(t) \\
& -\sum_{j=1}^{m} x^{T}\left(t-h_{j}\right) W_{j} x\left(t-h_{j}\right) \\
& -\sum_{j=1}^{m} \int_{-h_{j}}^{0} x^{T}(t+\theta) W_{m+j} x(t+\theta) d \theta \\
& -\int_{-\tau}^{0} x^{T}(t+\theta) M x(t+\theta) d \theta .
\end{aligned}
$$

Proof. Define in (13) the change of variable $\xi=t+\theta$ in both integrals, then

$$
\begin{aligned}
\tilde{v}\left(x_{t}\right)= & \sum_{j=1}^{m} \int_{t-h_{j}}^{t} x^{T}(\xi)\left[W_{j}+\left(h_{j}+\xi-t\right) W_{m+j}\right] \times \\
& x(\xi) d \xi+\int_{t-\tau}^{t}(\tau+\xi-t) x^{T}(\xi) M x(\xi) d \xi
\end{aligned}
$$

Differentiating with respect to $t$, yields

$$
\begin{aligned}
\frac{d \tilde{v}\left(x_{t}\right)}{d t}= & x^{T}(t)\left\{\sum_{j=1}^{m}\left(W_{j}+h_{j} W_{m+j}\right)+\tau M\right\} x(t) \\
& -\sum_{j=1}^{m} x^{T}\left(t-h_{j}\right) W_{j} x\left(t-h_{j}\right) \\
& -\sum_{j=1}^{m} \int_{-h_{j}}^{0} x^{T}(t+\theta) W_{m+j} x(t+\theta) d \theta \\
& -\int_{-\tau}^{0} x^{T}(t+\theta) M x(t+\theta) d \theta .
\end{aligned}
$$

Now, choose in the functional (12) the matrix $U(\tau)$ with $W$ defined as follows

$$
W=W_{0}+\sum_{j=1}^{m}\left(W_{j}+h_{j} W_{m+j}\right)+\tau M,
$$

and consider the sum

$$
v\left(x_{t}\right)=v_{0}\left(x_{t}\right)+\tilde{v}\left(x_{t}\right),
$$

the functional $v\left(x_{t}\right)$ is

$$
\begin{aligned}
v\left(x_{t}\right)= & x^{T}(t) U(0) x(t)+2 x^{T}(t) \times \\
& {\left[\sum_{j=1}^{m} \int_{-h_{j}}^{0} U\left(-h_{j}-\zeta\right) A_{j} x(t+\zeta) d \zeta+\right.} \\
& \left.\int_{-\tau}^{0} \int_{\theta}^{0} U(\theta-\zeta) Q(\theta) x(t+\zeta) d \zeta d \theta\right] \\
& +\sum_{j=1}^{m} \int_{-h_{j}}^{0}\left[\sum_{k=1}^{m} \int_{-h_{k}}^{0} x^{T}\left(t+\zeta_{1}\right) A_{k}^{T} \times\right. \\
& U\left(h_{k}+\zeta_{1}-h_{j}-\zeta_{2}\right) A_{j} x\left(t+\zeta_{2}\right) d \zeta_{1} \\
& +2 \int_{-\tau}^{0} \int_{\theta}^{0} x^{T}\left(t+\zeta_{1}\right) Q^{T}(\theta) \times \\
& \left.U\left(\zeta_{1}-\theta-h_{j}-\zeta_{2}\right) A_{j} x\left(t+\zeta_{2}\right) d \zeta_{1} d \theta\right] d \zeta_{2} \\
& +\int_{-\tau}^{0} \int_{\theta_{1}}^{0} \int_{-\tau}^{0} \int_{\theta_{2}}^{0} x^{T}\left(t+\zeta_{1}\right) Q^{T}\left(\theta_{1}\right) \times \\
& U\left(\zeta_{1}-\theta_{1}-\zeta_{2}+\theta_{2}\right) Q\left(\theta_{2}\right) x\left(t+\zeta_{2}\right) \times \\
& d \zeta_{2} d \theta_{2} d \zeta_{1} d \theta_{1}+\sum_{j=1}^{m} \int_{-h_{j}}^{0} x^{T}(t+\theta) \times \\
& {\left[W_{j}+\left(h_{j}+\theta\right) W_{m+j}\right] x(t+\theta) d \theta } \\
& +\int_{-\tau}^{0}(\tau+\theta) x^{T}(t+\theta) M x(t+\theta) d \theta .(17)
\end{aligned}
$$

and it follows of (10) and (14) that the time derivative of $v\left(x_{t}\right)$ along the trajectories of system (1) is $-w\left(x_{t}\right)$. We are now able to state our main result.

Theorem 2. Let system (1) be stable. Given the $n \times n$ definite positive matrices $W_{0}, W_{k}, W_{m+k}$, $M, k=1,2, \ldots, m$, the functional (17) satisfies the condition

$$
\frac{d}{d t} v\left(x_{t}\right)=-w\left(x_{t}\right) .
$$


Proof. The result can be verified by direct calculation.

Remark 1. It follows from (17) that the functional $v\left(x_{t}\right)$ is completely determined once the matrix function $U(\tau), \tau \in[0, H]$ is known. The numerical construction of $U(\tau)$ is an essential part of our current research. For the case of systems with distributed delays, one possible approache is the computation of piecewise linear approximations of $U(\tau)$, see (Garcia-L. and Kharitonov, 2004). Notice also that if matrix $W$, defined in (16), is given then the corresponding matrix function $U(\tau)$ is unique, while a great deal of freedom in the choice of the matrices $W_{0}, W_{k}, W_{m+k}, M$, $k=1,2, \ldots, m$ is still left.

Lemma 3. For some constant $\alpha>0$, the functional (17) admits a quadratic lower bound of the form

$$
\alpha\|x(t)\|^{2} \leq v\left(x_{t}\right) .
$$

Proof. Define the functional

$$
v^{(\alpha)}\left(x_{t}\right)=v\left(x_{t}\right)-\alpha\|x(t)\|^{2} .
$$

The time derivative of $v^{(\alpha)}\left(x_{t}\right)$ along the trajectories of the system (1) is

$$
\begin{aligned}
& \frac{d}{d t} v^{\left(\alpha_{1}\right)}\left(x_{t}\right)=-w^{\left(\alpha_{1}\right)}\left(x_{t}\right)=-w\left(x_{t}\right)-2 \alpha x^{T}(t) \times \\
& {\left[\sum_{k=0}^{m} A_{k} x\left(t-h_{k}\right)+\int_{-\tau}^{0} Q(\theta) x(t+\theta) d \theta\right]} \\
& =-x^{T}(t) W_{0} x(t)-\sum_{k=1}^{m} x^{T}\left(t-h_{k}\right) W_{k} x\left(t-h_{k}\right) \\
& -\sum_{k=1}^{m} \int_{-h_{k}}^{0} x^{T}(t+\theta) W_{m+k} x(t+\theta) d \theta \\
& -\int_{-\tau}^{0} x^{T}(t+\theta) M x(t+\theta) d \theta-2 \alpha x^{T}(t) \times \\
& {\left[\sum_{k=0}^{m} A_{k} x\left(t-h_{k}\right)+\int_{-\tau}^{0} Q(\theta) x(t+\theta) d \theta\right] .}
\end{aligned}
$$

It follows that

$$
\begin{aligned}
& w^{(\alpha)}\left(x_{t}\right) \leq\left[\begin{array}{c}
x(t) \\
x\left(t-h_{1}\right) \\
\vdots \\
x\left(t-h_{m}\right)
\end{array}\right]^{T} M(\alpha)\left[\begin{array}{c}
x(t) \\
x\left(t-h_{1}\right) \\
\vdots \\
x\left(t-h_{m}\right)
\end{array}\right] \\
& +\sum_{k=1}^{m} \int_{-h_{k}}^{0} x^{T}(t+\theta) W_{m+k} x(t+\theta) d \theta \\
& +\int_{-\tau}^{0} x^{T}(t+\theta) M x(t+\theta) d \theta \\
& -\alpha \int_{-\tau}^{0} x^{T}(t+\theta) Q^{T}(\theta+\tau) Q(\theta+\tau) x(t+\theta) d \theta .
\end{aligned}
$$

where

$$
M(\alpha)=\left[\begin{array}{cccc}
W_{0}+\alpha\left(A_{0}+A_{0}^{T}-I_{n}\right) & \alpha A_{1} & \cdots & \alpha A_{m} \\
\alpha A_{1}^{T} & W_{1} & \cdots & 0 \\
\vdots & \vdots & \ddots & \vdots \\
\alpha A_{m}^{T} & 0 & \cdots & W_{m}
\end{array}\right]
$$

Using the fact that each element of the matrix $Q(\theta)$ is continuous in $[-H, 0]$, we have that $\left\|Q^{T}(\theta) Q(\theta)\right\|_{H} \leq \bar{q}$, so we have that

$$
\begin{aligned}
w^{(\alpha)}\left(x_{t}\right) \leq & {\left[\begin{array}{c}
x(t) \\
x\left(t-h_{1}\right) \\
\vdots \\
x\left(t-h_{m}\right)
\end{array}\right]^{T} M(\alpha)\left[\begin{array}{c}
x(t) \\
x\left(t-h_{1}\right) \\
\vdots \\
x\left(t-h_{m}\right)
\end{array}\right] } \\
& +\sum_{k=1}^{m} \int_{-h_{k}}^{0} x^{T}(t+\theta) W_{m+k} x(t+\theta) d \theta \\
& +\int_{-\tau}^{0} x^{T}(t+\theta)\left(M-\alpha \bar{q} I_{n}\right) x(t+\theta) d \theta .
\end{aligned}
$$

Then there exists an $\alpha>0$ such that

$$
M(\alpha)>0 \text { and } M-\alpha \bar{q} I_{n}>0
$$

Indeed, as $W_{0}, W_{k}, W_{m+k}$ and $M, k=1,2, \ldots, m$, are positive definite matrices, we have that

$$
w^{(\alpha)}\left(x_{t}\right) \geq 0, \text { for all } t \geq 0 .
$$

hence

$$
v^{(\alpha)}(\varphi)=\int_{0}^{\infty} w^{(\alpha)}\left(x_{t}(\varphi)\right) d t \geq 0
$$

and it follows that

$$
\alpha\|x(t)\|^{2} \leq v\left(x_{t}\right) .
$$

Lemma 4. For some constant $\varepsilon>0$, the functional (17) admits the following quadratic upper bound

$$
\begin{aligned}
v\left(x_{t}\right) \leq & \varepsilon\left\{\|x(t)\|^{2}+\sum_{k=1}^{m} \int_{-h_{k}}^{0}\|x(t+\theta)\|^{2} d \theta\right. \\
& \left.+\int_{-\tau}^{0}\|x(t+\zeta)\|^{2} d \zeta\right\}
\end{aligned}
$$

Proof. Let

$$
\begin{aligned}
\nu & =\max \|U(\tau)\|, \tau \in[0, H], \\
a & =\max \left\|A_{1}\right\|, i=1,2, \ldots, m \\
\omega & =\max \left\{\left\|W_{1}\right\|,\left\|W_{1}\right\|, \ldots,\left\|W_{m}\right\|,\|M\|\right\}
\end{aligned}
$$

Using appropiate majorizations for each term of functional described by (17) leads to

$$
\begin{aligned}
v\left(x_{t}\right) \leq & \eta_{1}\|x(t)\|^{2}+\eta_{2} \sum_{k=1}^{m} \int_{-h_{k}}^{0}\|x(t+\theta)\|^{2} d \theta \\
& +\eta_{3} \int_{-\tau}^{0}\|x(t+\zeta)\|^{2} d \zeta
\end{aligned}
$$


where

$$
\begin{aligned}
& \eta_{1}=\nu+\frac{\tau^{2} q \nu}{2}+\nu a \sum_{j=1}^{m} h_{j} \\
& \eta_{2}=\nu a^{2} \sum_{j=1}^{m} h_{j}+a \nu+a \frac{\tau^{2} q \nu}{2}+\omega\left(1+h_{m}\right) \\
& \eta_{3}=q \nu \tau+\tau q \nu a \sum_{j=1}^{m} h_{j}+\frac{\tau^{3} q^{2} \nu}{2}+\omega \tau
\end{aligned}
$$

and the result holds for any $\varepsilon$ such that $\varepsilon \geq$ $\max \left\{\eta_{1}, \eta_{2}, \eta_{3}\right\}$.

Remark 2. Using (20), (21), (22) and the fact that $\|x(t)\| \leq\left\|x_{t}\right\|_{H}$, it is not difficult to prove that the functional (17) also satisfies

$$
v\left(x_{t}\right) \leq \alpha_{1}\left\|x_{t}\right\|_{H}^{2} .
$$

\section{EXPONENTIAL BOUNDS.}

In this section, for a given initial function, we obtain an exponential estimate of the form (2) of the solution of System (1) based on the complete type functional (17). First we prove a preliminary result.

Lemma 5. Let System (1) be exponentially stable. Given positive definite matrices $W_{0}, W_{k}$, $W_{m+k}$ and $M$, there exists a constant $\beta>0$, such that the functional $w\left(x_{t}\right)$ defined in (9) satisfies

$$
2 \beta v\left(x_{t}\right) \leq w\left(x_{t}\right) .
$$

Proof. Consider (23) and a lower bound for $w\left(x_{t}\right)$ obtained as follows. Let

$\mu=\min \left\{\lambda_{\min }\left(W_{j}\right), \lambda_{\min }(M)\right\}, j=0,1,2, \ldots, 2 m$, then

$$
\begin{gathered}
\mu\left(\|x(t)\|^{2}+\sum_{k=1}^{m} \int_{-h_{k}}^{0}\|x(t+\theta)\|^{2} d \theta\right. \\
\left.+\int_{-\tau}^{0}\|x(t+\zeta)\|^{2} d \zeta\right) \leq w\left(x_{t}\right) .
\end{gathered}
$$

Finally observe that if we choose $\beta$ as

$$
2 \beta=\min \left\{\frac{\mu}{\eta_{1}}, \frac{\mu}{\eta_{2}}, \frac{\mu}{\eta_{3}}\right\}
$$

the expression (25) is satisfied.

Theorem 3. Let system (1) be exponentially stable and the matrices $W_{i}, i=1, \ldots, 2 m$, be given. For any initial condition $\varphi(\theta), \theta \in[-H, 0]$, the solution of system (1) satisfies the inequality

$$
\|x(t, \varphi)\| \leq \sqrt{\frac{\alpha_{1}}{\alpha}}\|\varphi\|_{H} e^{-\beta t}, \quad t \geq 0,
$$

where $\alpha, \alpha_{1}$ are the constants defined by (18) and (24), respectively, and $\beta$ is defined in (26).
Proof. Observe that (25) implies that

$$
\frac{d v\left(x_{t}\right)}{d t} \leq-w\left(x_{t}\right) \leq-2 \beta v\left(x_{t}\right),
$$

solving for $v\left(x_{t}\right)$ yields $v\left(x_{t}\right) \leq e^{-2 \beta t} v(\varphi)$. Then, (3) and (24), imply that

$\alpha\|x(t, \varphi)\|^{2} \leq v\left(x_{t}\right) \leq v(\varphi) e^{-2 \beta t} \leq \alpha_{1} e^{-2 \beta t}\|\varphi\|_{H}^{2}$, and the result follows from the first and last terms of these inequalities.

\section{ROBUST STABILITY}

In this section we show that the complete type functional can be used to derive robust stability conditions. Consider the perturbed system

$$
\begin{aligned}
\dot{y}(t)= & \sum_{k=0}^{m}\left(A_{k}+\Delta_{k}\right) y\left(t-h_{k}\right) \\
& +\int_{-\tau}^{0}(Q(\theta)+\Delta(\theta)) y(t+\theta) d \theta .
\end{aligned}
$$

where

$$
\begin{aligned}
\left\|\Delta_{k}\right\| & \leq \rho_{k}, \quad k=0,1,2, \ldots, m . \\
\|\Delta(\theta)\|_{\tau} \leq \delta_{q}, & \theta \in[-\tau, 0] .
\end{aligned}
$$

Let the nominal system described by (1) be stable. Using the complete type functional constructed in Section 3, we want to derive conditions under which the perturbed system remains stable. The derivative of $v\left(y_{t}\right)$ along the trajectories of $(27)$ is

$$
\begin{aligned}
& \left.\frac{d v\left(y_{t}\right)}{d t}\right|_{(27)}=-w\left(y_{t}\right) \\
& +2\left[\sum_{k=0}^{m} \Delta_{k} y\left(t-h_{k}\right)+\int_{-\tau}^{0} \Delta(\theta) y(t+\theta) d \theta\right]^{T} \\
& {\left[U(0) y(t)+\sum_{j=1}^{m} \int_{-h_{j}}^{0} U\left(-h_{j}-\zeta\right) A_{j} y(t+\zeta) d \zeta\right.} \\
& \left.+\int_{-\tau}^{0} \int_{\theta}^{0} U^{T}(\theta-\zeta) Q(\theta) y(t+\zeta) d \zeta d \theta\right] .
\end{aligned}
$$

Considering (20), (28) and (21) we thus see that

$$
\begin{aligned}
& \left.\frac{d v\left(y_{t}\right)}{d t}\right|_{(27)} \leq-y^{T}(t)\left\{W_{0}-\Gamma_{1} I_{n}\right\} y(t) \\
& -\sum_{k=1}^{m} y^{T}\left(t-h_{k}\right)\left\{W_{k}-\Gamma_{2} I_{n}\right\} y\left(t-h_{k}\right) \\
& -\sum_{k=1}^{m} \int_{-h_{k}}^{0} y^{T}(t+\zeta)\left\{R_{k}-\Gamma_{3} I_{n}\right\} y(t+\zeta) d \zeta \\
& -\int_{-\tau}^{0} y^{T}(t+\theta)\left\{M-\Gamma_{4} I_{n}\right\} y(t+\theta) d \theta
\end{aligned}
$$

where 


$$
\begin{aligned}
\Gamma_{1}= & 2 \nu \rho_{0}+\sum_{k=1}^{m} \rho_{k} \nu+\nu \delta_{q} \tau+\rho_{0} \nu a \sum_{j=1}^{m} h_{j} \\
& +\nu \rho_{0} q_{1} \frac{\tau^{2}}{2} \\
\Gamma_{2}= & \rho_{k} \nu+a \nu \sum_{j=1}^{m} \rho_{k} h_{j}+\nu q_{1} \frac{\tau^{2}}{2} \rho_{k} \\
\Gamma_{3}= & \nu a \sum_{j=0}^{m} \rho_{j}+\delta_{q} \nu \tau a \\
\Gamma_{4}= & \nu \delta_{q}+\tau \nu q_{1} \sum_{k=0}^{m} \rho_{k}+\frac{3}{2} \tau^{2} \delta_{q} \nu q_{1}
\end{aligned}
$$

From the above, we can write the following result.

Theorem 4. Let System (1) be stable. Then the system (27) remains stable for all perturbations which satisfy the conditions (28) if there exist positive definite matrices $W_{0}, W_{k}, R_{k}$ and $M$, $k=1,2, . ., m$ such that

$W_{0}>\Gamma_{1} I_{n}, \quad W_{k}>\Gamma_{2} I_{n}, R_{k}>\Gamma_{3} I_{n}, \quad M>\Gamma_{4} I_{n}$

\section{CONCLUSIONS}

In this contribution, the construction of complete type functionals proposed in (Kharitonov, 2003) is extended to systems with distributed time delays. Quadratic lower and upper bounds for these functionals are obtained. It is shown that these functionals can be employed to determine exponential bounds for the solutions of systems with distributed time delays and to find conditions of robust stability when there is uncertainty in the parameters.

\section{REFERENCES}

Bellman, R. and K. Cooke (1963). Differential Difference Equations. Academic Press.

Datko, R. (1971). An Algorithm for Computing Lyapunov Functionals for some Differential Difference Equations. In L. Weiss. Academic Press (p. 387-398).

Garcia-L., H. and V. L. Kharitonov (2004). Lyapunov matrices for time delay system with commensurate delays. 2nd IFAC Symp. on System, Structure and Control, 102-106.

Huang, Wenzhang (1989). Generalization of lyapunov's theorem in a linear delay system. J. of Mathematical analysis and applications 142, 83-94.

Infante, E. F. Y Castelan, W. B. (1978). A lyapunov functional for a matrix difference differential equation. Int. J. of control 29, 439451.

Kharitonov, V.L., A.P. Zhabko (2003). Lyapunovkrasovskii approach to the robust stability analisys of time delay systems. Automatica 39, 15-20.
Repin, Yu. M. (1965). Quadratic lyapunov functionals for systems with delay.. Prikladnay Matematika Mekhanika 29, 564-566. 